\section{Cahiers balkaniques}

42 | 2014

Grèce-Roumanie : héritages communs, regards croisés

\title{
Le Cheval de Moldovalachie de Georges Séféris
}

The Horse of Moldovlahia, Georges Seferis

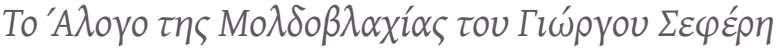

\section{Georges Kostakiotis}

\section{OpenEdition}

Journals

Édition électronique

URL : https://journals.openedition.org/ceb/4880

DOI : $10.4000 /$ ceb.4880

ISSN : 2261-4184

Éditeur

INALCO

\section{Référence électronique}

Georges Kostakiotis, «Le Cheval de Moldovalachie de Georges Séféris », Cahiers balkaniques [En ligne], 42 | 2014, mis en ligne le 27 mai 2014, consulté le 07 juillet 2021. URL : http:// journals.openedition.org/ceb/4880 ; DOI : https://doi.org/10.4000/ceb.4880

Ce document a été généré automatiquement le 7 juillet 2021.

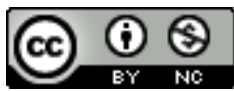

Cahiers balkaniques est mis à disposition selon les termes de la Licence Creative Commons Attribution - Pas d'Utilisation Commerciale 4.0 International. 


\title{
Le Cheval de Moldovalachie de Georges Séféris
}

\author{
The Horse of Moldovlahia, Georges Seferis

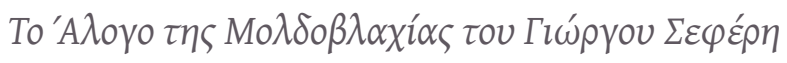

\section{Georges Kostakiotis}

1 Georges Séféris, durant l'année 1939, en tant que directeur du Bureau de Presse du ministère des Affaires étrangères de Grèce, a effectué deux visites en Roumanie; la première du 20 au 24 février, certainement une visite officielle, le poète faisant partie de la mission grecque accompagnant le Premier ministre, Ioannis Metaxas, - qui avait instauré une dictature depuis 1936 - , pour la réunion du Conseil de l'Entente balkanique, la seconde deux mois plus tard, du 14 ou 29 mai.

2 Le Cheval de Moldovalachie est le poème que Georges Séféris a composé durant son deuxième séjour à Bucarest. Il s'agit d'une composition plutôt longue (si on la compare aux autres poèmes) et qui n'a jamais fait partie d'aucun recueil publié par le poète de son vivant. Il l'a lui-même qualifiée d'esquisse qui, en tant que telle, fait partie de son journal intime daté du 19 mai 1939. Ce poème a également été publié en 1976, après la mort du poète, avec certaines variations, sans la mention esquisse, mais toujours daté du

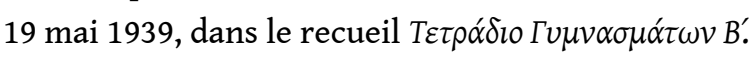

3 Le poète, influencé par le climat angoissant du début de la Seconde Guerre mondiale, transpose ses sentiments négatifs sur la situation politique décadente durant ses séjours en Roumanie et notamment à Bucarest.

4 Georges Séféris, depuis sa fenêtre de l'Athénée Palace dominant le palais royal et la Calea Victoriei, est comme fasciné par la statue équestre du roi Carol I ${ }^{\text {er }}$ qui vient d'être inaugurée - justement le 10 mai 1939 -, à l'occasion de la célébration du centenaire de sa naissance en $1839^{1}$, pour célébrer l'avènement du premier roi au trône le 10 mai 1866.

5 Cette statue a vraisemblablement inspiré cette esquisse. Nous nous trouvons au centre politique de la capitale roumaine, au cœur de la ville où symboliquement le pouvoir actuel affronte l'histoire du pays. La statue du roi et le roi dans le palais s'opposent et 
s'affrontent jusqu'à la confusion. La réalité face à l'histoire semble fuir ses responsabilités.

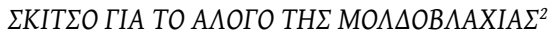

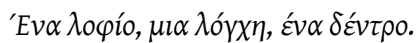

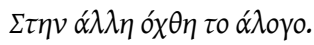

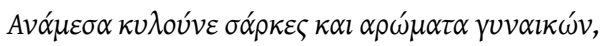

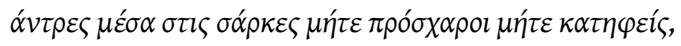

$\alpha \pi \circ \varphi \alpha \sigma \iota \sigma \mu \varepsilon ́ v o l$,

óхl $\alpha \pi \circ \varphi \alpha \sigma l \sigma \tau \imath \kappa o i ́$,

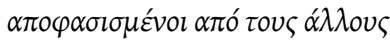

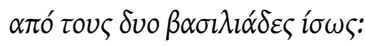

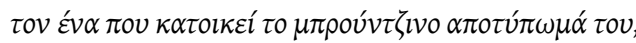

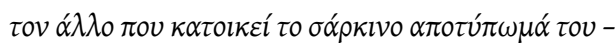

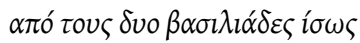

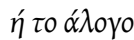

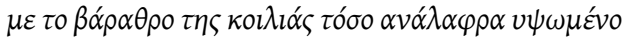

$\pi \alpha \dot{\alpha} v \omega \sigma \tau \alpha \tau \varepsilon \dot{\varepsilon} \sigma \sigma \varepsilon \rho \alpha \pi \delta_{\delta} \alpha$

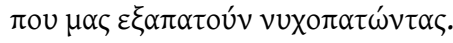

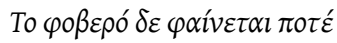

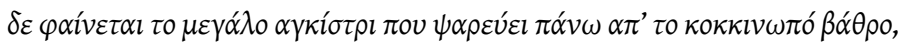

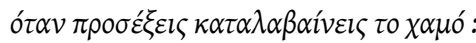

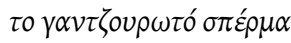

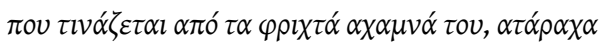

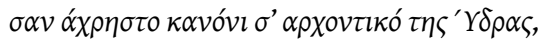

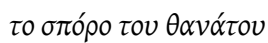

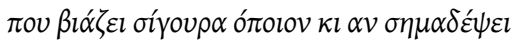

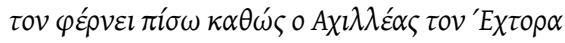

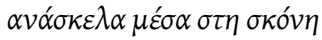

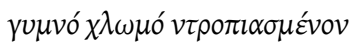

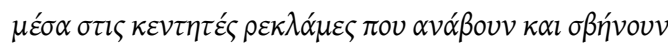

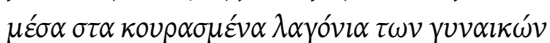

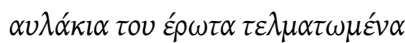

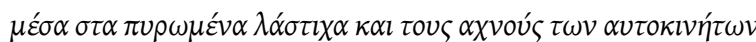

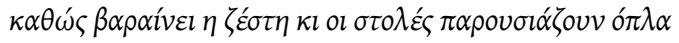

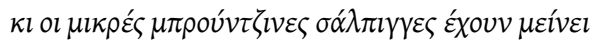

$\alpha \nu \alpha ́ \varepsilon \rho \varepsilon \varsigma-$

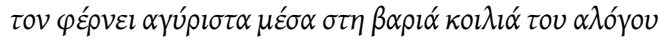

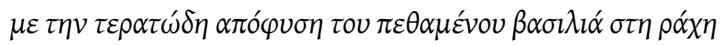

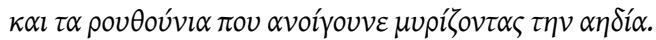

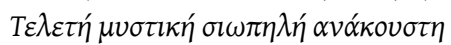

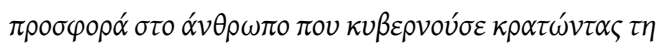

$\sigma \varphi \alpha i \rho \alpha \kappa \alpha l \tau o \sigma \kappa \eta ́ \pi \tau \rho o$

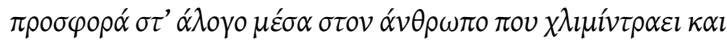

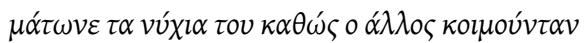

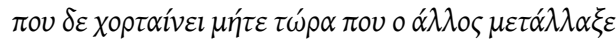

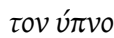

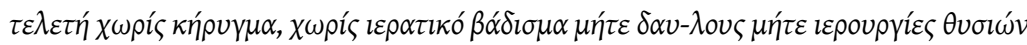

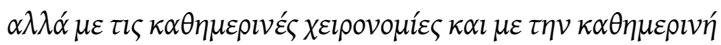

$\mu \alpha \varsigma \sigma v \mu \pi \varepsilon \rho l \varphi \circ \rho \alpha ́$

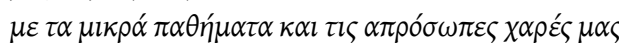

$\mu \varepsilon \tau \eta \sigma v v \eta \theta l \sigma \mu \varepsilon \dot{v} \eta \eta$

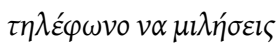

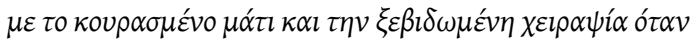

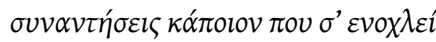




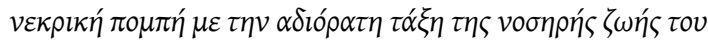

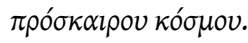

ESQUISSE POUR LE CHEVAL DE MOLDOVALACHIE

Un panache, une lance, un arbre.

Sur l'autre rive, un cheval.

Au milieu coulent des chairs et des parfums de femmes,

dans ces chairs, des hommes ni joyeux ni maussades,

décidés,

pas décideurs,

décidés par le fait d'autrui ;

peut-être par ces deux rois :

l'un habitant son empreinte de bronze

l'autre habitant son empreinte de chair

par les deux rois peut-être

ou par le cheval

au ventre-gouffre si légèrement porté

sur quatre jambes

qui nous dupent sur la pointe des pieds.

L'horreur n'est jamais visible

on ne voit pas le grand hameçon qui pêche par-dessus

le piédestal rougeâtre,

quand on fait attention, on comprend la catastrophe :

le sperme crochu

qui jaillit de ses attributs terribles, impassibles,

tel un canon inutile dans un manoir d'Hydra,

semence de mort

qui viole sûrement quiconque il vise ;

le traîne comme Achille, traînait Hector

le dos au sol dans la poussière

nu pâle déshonoré

parmi les réclames brodées qui clignotent

dans le flanc fatigué des femmes

sillons embourbés de l'amour

au milieu des pneus chauffés à blanc et des exhalaisons

des automobiles

alors que la chaleur accable et que les uniformes présentent

leurs armes et que les petites trompettes de bronze sont restées

sans air -

il le porte inexorablement dans le lourd ventre du cheval

à l'appendice monstrueux du roi mort dans le dos

et les narines qui s'ouvrent sentant le dégoût.

Cérémonie secrète silencieuse inaudible

offrande à l'homme qui gouvernait tenant le

globe et le sceptre

offrande au cheval dans l'homme qui hennissait et

s'ensanglantait les ongles tandis que l'autre dormait

qui ne se rassasie même pas en ce moment où l'autre a transmuté

le sommeil

cérémonie sans sermon, sans démarche hiératique, ni flambeaux ni célébration de sacrifices,

mais avec nos gestes quotidiens et notre attitude

quotidienne

avec nos petites souffrances et nos joies impersonnelles

avec la ride habituelle au front quand on décroche le

téléphone pour parler

avec l'œil fatigué et la poignée de main molle quand on 
rencontre quelqu'un qui importune

cortège funèbre à l'ordre imperceptible de la vie maladive

du monde éphémère.

6 Nous essaierons d'étudier ce texte en développant ce que les symboles proposés par l'auteur semblent nous dire, ce sur quoi ils semblent nous alerter peut-être, nous interpeller sûrement, car le poème est souvent l'œuvre d'un visionnaire qui parvient à rendre compte de la réalité plus efficacement qu'un reportage ou qu'une description analysée et précise.

7 La connaissance poétique ne peut fonctionner qu'à travers l'expérience commune et universelle. Le sens que nous pouvons envisager dans ce travail de Séféris ne doit pas se limiter au niveau cognitif, il peut aussi se révéler à partir d'une relation qui traverse les situations émotionnelles. Pour arriver à cette connaissance, le poète nous invite à s'y adonner entièrement et à vivre avec l'esprit et les sens nourris de ces sensations ${ }^{3}$. La poésie n'atteint pas son but s'il n'y a pas concordance entre la sensibilité et le verbe poétique ; elle est obtenue uniquement s'il y a une certaine objectivité dans l'expression de l'émotion ${ }^{4}$ due au partage.

8 Pour une plus grande clarté du propos nous retiendrons quatre thèmes symboliquement présents dans le texte de Séféris: le Cheval, Bucarest et plus amplement la Roumanie, la Grèce et enfin le Roi.

\section{Le Cheval}

9 Chacun sait que le cheval est la plus noble conquête de l'homme, mais nous voyons ici une inversion du sens de la domination; dans cette statue équestre, le roi n'est plus qu'un "appendice ", l'image du cheval dans la poésie séférienne devient le symbole d'un danger imminent. Emballés, pleins d'écume ${ }^{5}$ et en sueur ${ }^{6}$ ou bien en marche lente et lourde ${ }^{7}$, les chevaux sont ressentis par le poète comme une force extérieure que l'homme n'arrive pas à contrôler. Ils incarnent le destin inconnu et insaisissable qui piétine l'homme ${ }^{8}$. De la même façon dans le recueil chypriote, Les Trois Mules expriment la mort violente ${ }^{9}$. C'est dans ce même esprit que doit être vu le cheval dans l'œuvre que nous étudions. Mentionné dans le titre du poème, l'animal est omniprésent dans l'ensemble de la composition ${ }^{10}$.

10 La catastrophe, la perte, l'anéantissement menacent, comme le cheval du haut de son piédestal. Cette ambiance moribonde pèse sur la ville, les trompes (symbole de l'Apocalypse) de la garde royale ne peuvent plus transmettre aucun message alors que les armes se présentent ${ }^{11}$. Le cheval "sent» le dégoût, le roi n'est réduit qu'à un « appendice monstrueux ». Le cheval devient un symbole à double signification, d'une part la tromperie, la ruse et en fin de compte la guerre qui hantent la ville, la capitale, l'Europe et donc le monde, d'autre part l'histoire et le passé qui ne se retrouvent plus, ne se reconnaissent plus, dans ce monde écœurant qui donne la nausée.

11 Sous le cheval, le socle, le piédestal fait partie de ce qui inspire le poète : avec la volonté

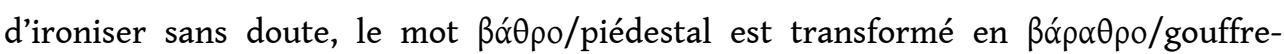
abîme. Le ventre du cheval désormais associé à l'abîme, contraste avec sa position «si légèrement soulevé(e) $»^{12}$ depuis son piédestal. Au-dessus est suspendu l'hameçon invisible, autre signe de tromperie et de ruse. C'est devant cette tromperie que le poète paraît exaspéré et crie à la vigilance, en soulignant que "l'horreur n'est jamais visible $»^{13}$. Cette exclamation renvoie aussi à une vérité nettement plus prosaïque : en 
effet, durant la première visite que Georges Séféris a effectuée en Roumanie, la statue était sous échafaudages, non inaugurée. Il n'en a discerné que les sabots - selon le témoignage, en $1961^{14}$, de Takis Papatzonis qui accompagnait le poète ${ }^{15}$ - et non pas le cheval entier. Ainsi nous pouvons comprendre la concentration, sinon la fixation du regard et de l'attention du poète sur le socle, les pattes et sur le ventre qu'il a probablement aperçus en regardant par en dessous.

Dans cette perception, la semence qui est une image de la vie, de la création et de la naissance se transforme ici en graine qui génère la mort, en opposition à la «graine qui meurt » pour redonner vie, image de l'Évangile qui sera reprise par Séféris plus tard pour exprimer son optimisme ${ }^{16}$.

13 Si à cette image nous ajoutons celle de la statue - puisqu'il s'agit d'une statue équestre le symbole prend une deuxième signification. Nous nous trouvons devant le vide ou l'impasse, la rupture avec le passé, l'absence d'avenir. Rappelons que cette image des statues ou encore des chevaux associés aux statues brisées est chère à Séféris et constamment présente dans son œuvre ${ }^{17}$. Il va sans dire que le cheval est ici associé à la guerre qui a déjà éclaté.

\section{Bucarest, la Roumanie}

14 À travers cette statue équestre, bien réelle, bien existante, le poète nous place au centre de Bucarest. Ainsi la menace de la guerre qu'elle évoque et qui est ressentie par le poète, se déplace au centre de la capitale qui, étant le lieu du pouvoir, devient le centre de l'Europe.

Nous sommes dans un lieu de pouvoir, mais de quel type de pouvoir? Le poète oppose la cité antique, comme lieu de démocratie, de discussions et de prise de décisions issues de dialogues, aux systèmes monarchiques, dictatoriaux. Nous sommes ici dans une société de Cyclopes - note-t-il dans son Journal, quelques jours auparavant - en citant l'Odyssée : Chez eux, pas d'assemblée qui juge ou délibère; mais, au haut des grands monts, au creux de sa caverne, chacun, sans s'occuper d'autrui, dicte sa loi à ses enfants et femmes ${ }^{18}$.

La Roumanie, à l'occasion de la réunion du conseil des Balkans, devient pour le poète l'expression de l'Europe décadente. Bucarest est la scène politique où l'histoire, l'héritage européen, se trouvent face à l'absolutisme du pouvoir aveugle qui, incapable de gouverner, de réagir ou de comprendre, sombre vers le néant, la mort.

17 Entre la statue et le palais, la rue de Bucarest la plus centrale et aussi la plus ancienne, la plus chargée historiquement, l'emblématique Calea Victoriei, incarne à elle seule l'histoire de Bucarest et l'unité de la Roumanie. Cette rue dont le nom commémore la Victoire du roi Carol $I^{\text {er }}$ face aux Turcs se transforme en un piège, en un espace de mort $^{19}$. Les gens, anéantis, victimes, mais aussi responsables, manquent de $v^{20}{ }^{20}$. Incapables de décider, "non décideurs ", ils laissent les autres le faire à leur place ${ }^{21}$. Le peuple, le monde entier, sans volonté, sans sentiment, semblent pris au piège d'une situation qui leur échappe. La vie n'est qu'un souvenir lointain comme l'odeur du parfum d'une femme qui n'est plus là : « des chairs et des parfums de femmes ${ }^{22}$.

18 Les enseignes des magasins qui clignotent, l'amour décadent à 40 lei, dira Séféris un peu plus loin ${ }^{23}$, la chaleur, les pneus et les « exhalaisons des voitures » constituent un monde pathétique ${ }^{24}$, une ville déshumanisée où «l'homme est mort, entraîné le dos au

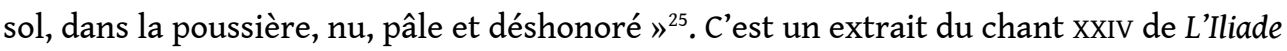


que le poète cite, rappelant Achille et Hector, pour nous donner une des images les plus fortes de la littérature antique. En même temps, il nous fait descendre dans les Enfers comme le fait Homère, au chant xi de L'Odyssée cette fois-ci. Bien qu'à la version du poème publiée sans le contexte du journal, Séféris note explicitement le verbe

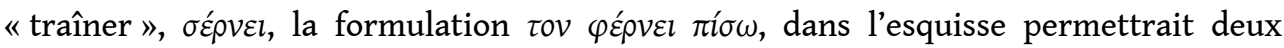
interprétations possibles : "il le traîne » et "il le fait revenir ». Le poète relie L'Iliade à L'Odyssée et retrouve les valeurs humanistes universelles pour faire face « au monde éphémère $»^{26}$ qu'il a trouvé à Bucarest, "aux capitales du grand monde où toute émotion s'évapore", notera-t-il plus tard ${ }^{27}$. Séféris se retourne vers le monde homérique, ne serait-ce qu'à travers les scènes les plus violentes, par opposition au monde actuel décadent et inerte.

\section{La Grèce}

La Grèce du poète subit le même sort. Séféris, à travers l'image du manoir et du canon de l'île d'Hydra (' $Y \delta \rho \alpha)$, la place également au centre du problème. Le nom ' $Y \delta \rho \alpha$, avec son orthographe, avec la voyelle $Y$ en majuscule, et sa prononciation due aux consonnes $\delta$ et $\rho$, est une allusion au monde grec et permet au poète de donner une image visuelle et phonétique. L'île, symbole de la lutte grecque contre l'occupant turc, devient un trait d'union entre la Grèce et la Roumanie et elle est utilisée par le poète pour faire le parallèle entre les deux pays à travers leur histoire commune dans leur combat pour l'indépendance jusqu'à leur reconnaissance par les Grandes Puissances. Leurs destins unis dans le passé semblent encore se croiser devant la guerre qui a déjà commencé. Seulement, cette fois-ci, le canon d'Hydra ne tonne plus, il devient

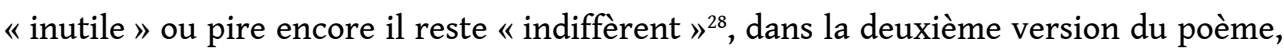
devant le germe de la mort qui se propage partout. Le poète montre ouvertement sa déception face à la position de la Grèce devant la situation en Europe : le monde grec semble incapable d'inspirer les valeurs de l'humanisme et de la démocratie. Enfin, notons que son nom, ' $Y \delta \rho \alpha$, fait allusion à $v \delta \rho i ́ \alpha$, la cruche en grec ancien, qui fonctionne comme une passerelle faisant la liaison entre monde actuel et le monde antique.

Malgré cette présence grecque au cœur du poème, Séféris ne reste pas enfermé dans le monde hellénique, comme il ne le reste d'ailleurs pas dans un cadre strictement roumain. Le but de la composition n'est ni grec, ni roumain, ni même balkanique ${ }^{29}$. Les symboles grecs trouvent leur place dans cette œuvre pour donner une dimension humaniste qui embrasse, à travers la capitale roumaine, toute la tradition européenne. Par conséquent, Bucarest devient le cœur par excellence du monde occidental; malheureusement il s'agit d'une Europe décadente et mourante gouvernée par des dictatures.

21 Les images de la réalité se conjuguent avec des images créées dans l'imagination du poète permettant une composition où le monde de son époque se heurte à son passé ; une fois de plus le présent n'est pas à la hauteur de son histoire qui, à son tour, devient insupportable et hante le quotidien. 


\section{Le Roi}

Le poète finit par nous placer dans le palais même en se souvenant de sa réception officielle par le roi lors de sa première visite, fin février. L'extrait de son journal qui décrit la réception est repris dans le poème. L'expérience du poète devient l'expérience du lecteur qui est invité au palais, à suivre de près les hommes politiques et à devenir le témoin d'un monde qui se contente de cérémonies sans message politique, creuses: " cérémonie sans sermon, sans démarche hiératique, ni flambeau, ni célébration de sacrifices ». L'ambiance moribonde de la rue se prolonge jusqu'au siège du pouvoir.

Le roi comme expression de ce pouvoir paraît déjà aux yeux du poète, dans son journal intime, comme "un docker travaillant au port $»^{30}$, rappelons que Séféris est arrivé à Bucarest depuis le port de Constantza. Entre «les deux rois », le roi Carol II et le roi Carol I ${ }^{\text {er }}$, le poète ressent l'impasse politique, il exprime son désaveu et exerce une critique vive envers les pouvoirs, les hommes de pouvoir et envers le système politique.

Séféris va même jusqu'à traiter le roi d'idiot; le propos prend une signification beaucoup plus forte et symbolique puisqu'il est mentionné comme une réflexion provenant d'un journaliste et homme politique turc ${ }^{31}$, symboliquement de l'envahisseur que Carol $\mathrm{I}^{\mathrm{er}}$ a vaincu pour asseoir l'indépendance du pays. Quant à sa place dans le poème, il semble être dans un état de "sommeil », une situation qui est associée chez Séféris à la mort et à la maladie. Il souligne d'ailleurs à propos de la statue qu'il s'agit du roi « mort », utilisant l'adjectif vekpós, ou bien, dans la version de l'esquisse, le participe passif $\pi \varepsilon \theta \alpha \mu \varepsilon ́ v o \varsigma$ comme pour mettre l'accent sur le caractère permanent de la situation.

Il note quelques jours auparavant que «nous vivons dans une époque de somnambulisme général $\aleph^{32}$, cette idée est reprise également à la fin du poème : le cérémonial finit par être un "cortège funèbre » dans une "vie maladive ", dans un "monde précaire $"^{33}$, dont la fin semble annoncée, pour lui conférer dimension de la fragilité, ou d'« éphémère "; il s'agit d'un monde qui annonce sa destruction. Dans la variante publiée du poème, dans le recueil $\Gamma v \mu v o \pi \alpha \_\delta i \alpha B^{\prime}$, le "monde éphémère/

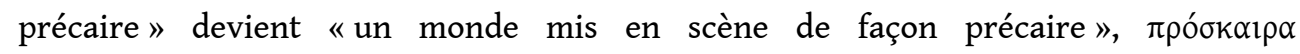

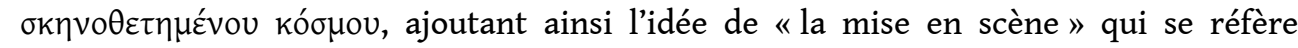
directement au Palais. Dans cette ambiance, nous retrouvons également le panache du début du poème qui semble être à sa juste place dans la réception-« mise en scène » par le roi. Ce symbole de la royauté décadente donne un ton ironique qui permet de décrier non seulement un système politique, mais aussi une situation qui se contente de l'application du protocole et de cérémonies d'apparat ${ }^{34}$.

Une fois de plus la satire devient une arme qui permet au poète d'exister, elle revient chaque fois que le ton monte et permet d'échapper au danger et de survivre. Selon Savidis, Le Cheval de Moldovalachie constitue une œuvre par excellence satirique et, de ce fait, une de meilleures compositions du Séféris d'avant-guerre ${ }^{35}$. Le poète essaie d'obtenir un équilibre entre les éléments réalistes et symboliques qu'il utilise en composant l'actualité du sujet avec la présentation suggestive des détails (les attributs $\mathrm{du}$ cheval, le ventre). Séféris, très certainement, a recours à de telles techniques par souci d'échapper au risque de considérer comme étant de la prose l'usage du vers libre. C'est pour cette même raison qu'il développe sa construction autour de répétitions 
rythmiques ${ }^{36}$. Ces répétitions donnent également le sentiment d'une action qui entraîne un mouvement, mais qui finalement est freinée par le sens des mots.

Rappelons qu'à cette même époque Séféris est en train de composer l'un des poèmes les plus significatifs : Le Roi d'Assine ; il note dans son journal qu'il y pense justement durant la réception au palais ${ }^{37}$. Nous y retrouvons certaines similitudes du point de vue technique et stylistique.

L'emploi du terme "esquisse " pour Le Cheval de Moldovalachie, nous permet de le considérer comme une ébauche pour Le Roi d'Assine. L'esquisse est un lieu d'essais, de contrôle des images, telles des poses (photographiques), pour la mise en place du « Roi d'Assine». Si la composition du «Cheval de Moldovalachie» ne semble pas très «installée », si elle semble être le théâtre de mouvements multiples, si elle représente le lieu même de la création, c'est qu'elle contient déjà d'importants éléments du "Roi d'Assine ». Les deux œuvres se lisent en parallèle et se complètent. Sans utiliser nécessairement la même architecture ni le même cadre, l'absence d'avenir, la rupture avec le passé et la décadence préoccupent le poète. Notons également la présence de l'image du roi ainsi que celle de la statue et du masque qui devient 4 empreinte $»^{38}$ dans Le Cheval de Moldovalachie; le monde d'Homère y est également évoqué.

Les mots sont ceux du quotidien et la description très réaliste et parfois crue; le ton émotionnel exprimé à travers l'ironie, associé à l'immédiateté expressive, crée une dissonance harmonieuse en accord parfait avec la déconstruction/ dissociation sentimentale ${ }^{39}$, voulue par le poète.

\section{Conclusion}

En guise de conclusion, nous voudrions revenir au Journal intime, aux pages qui précèdent la rédaction de l'esquisse, pour souligner la volonté du poète de jeter un regard optimiste malgré cette situation négative. Séféris, comme s'il voulait introduire le poème pour conjurer le Mal qui hante et envahit le monde, fait une brève évocation de sa visite au monastère de Vacarești, seul monument de la capitale mentionné avec la statue et le palais dans le journal intime. Deux inscriptions notées et citées marquent de manière importante leur lien avec le Cheval de Moldovalachie. Il s'agit de la source d'eau fraîche qui invite les fidèles à se désaltérer ainsi que de la pierre tombale dont l'épigraphe fait allusion à la mort sereine. Ces deux références s'opposent à une vie superficielle, d'apparat, de vanité, représentée par le Palais; elles accentuent la gravité de la situation et soulignent le déclin du monde occidental et de ses systèmes politiques.

Dans la même optique, l'acrostiche de Jean Cocteau (cité juste avant) qui forme le mot M.E.R.D.E, c'est-à-dire "Madame Eurydice reviendra des enfers" (cité en français), fonctionne comme une vraie partie du poème; il devient un appel à la vie, nous assurant de la résurrection à venir.

Malheureusement, sur un ton beaucoup plus sarcastique, Séféris notera dans un poème qui va suivre quelques jours plus tard que Le cheval n'a pas dit M.E.R.D.E. La résurrection n'a pas eu lieu. 


\section{BIBLIOGRAPHIE}

Durandin Catherine, (1995), Histoire des Roumains, Paris : Fayard.

Kohler Denis, (1985), L'Aviron d'Ulysse, Paris : Les Belles Lettres.

Morand Paul, (1935), Bucarest, Paris : Plon.

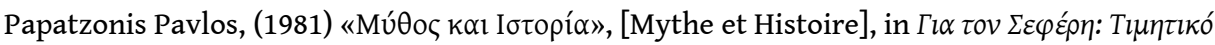

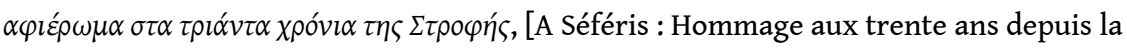
composition de Strophé], Athènes : Ermis, 1981 (1961), p. 24-31.

Séféris Georges, (1992), Пoı́́ $\alpha \alpha \tau \alpha$, [Poèmes], Athènes : Ikaros.

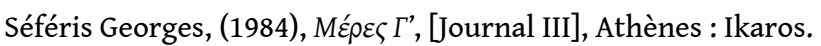

Séféris Georges, (2002), Journal de Bord III, édition commentée par Papazoglou Christos, Paris : Publications Langues' 0.

Savidis Giorgos, (1996) «Гı

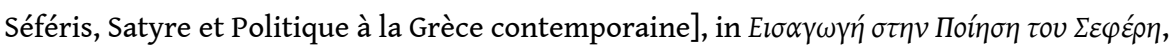
[Introduction à la poésie de Séféris], Héraklion : Panapistimiakes Ekdoseis Kritis, p. 307-340.

Tsirkas Stratis, (1961), «Mı $\alpha$ 'A

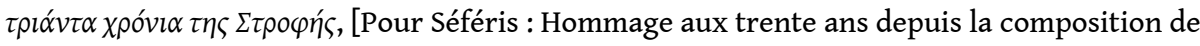
Strophé, Athènes : Ermis, (1961), p. 241-349.

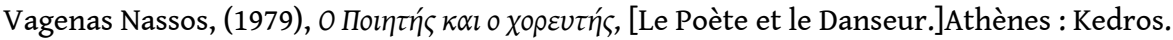

\section{NOTES}

1. La statue équestre du roi Carol I ${ }^{\mathrm{er}}$ a été démontée en 1947 et, selon une version, a été fondue pour ériger en 1960 la statue en l'honneur de Lénine qui se trouvait jusqu'en 1990 devant la Maison de la Presse. La statue équestre du roi Carol I ${ }^{\text {er }}$ qui se trouve actuellement devant la Bibliothèque Universitaire, face aux Galeries Nationales d'art, est une copie.

2. La traduction qui suit ne se veut pas un travail littéraire fini, mais une esquisse de traduction pour permettre la compréhension des commentaires proposés.

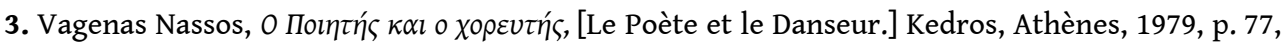
78.

4. Vagenas, 1979, op. cit., p. 53.

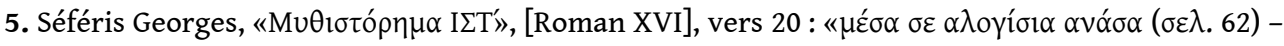

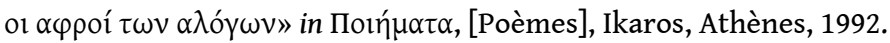

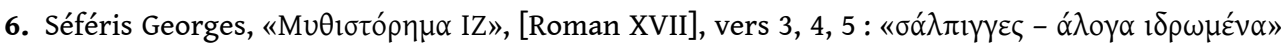
in Пoıń $\mu \alpha \tau \alpha$, [Poèmes], Ikaros, Athènes, 1992.

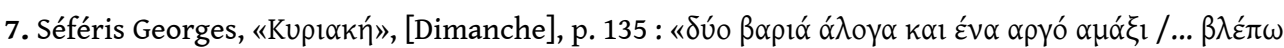

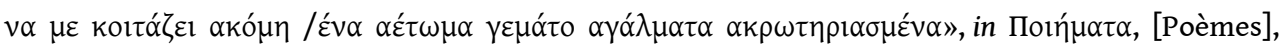
Ikaros, Athènes, 1992.

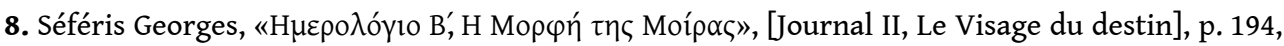

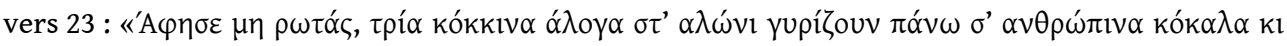

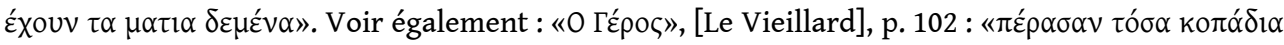

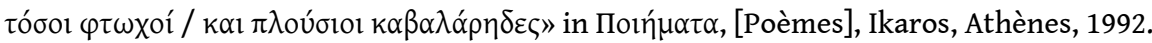


9. Séféris Georges, Journal de Bord III, édition commentée par Papazoglou Christos, Publications Langues' O, Paris, 2002, p. 246.

10. Sur le «cheval» voir aussi le commentaire de Kohler Denis, L'Aviron d'Ulysse, Les Belles Lettres, Paris, 1985, p. 582, 583.

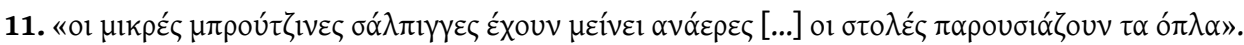

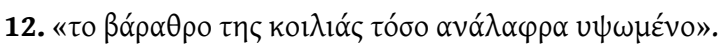

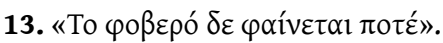

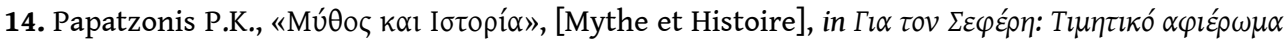

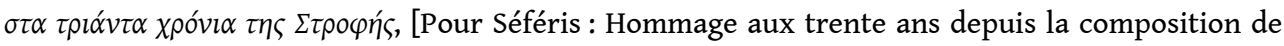
Strophé], Ermis, Athènes, 1981 (1961), p. 24-31.

15. Séféris a écrit un deuxième poème s'inspirant de cette même statue et des propos que

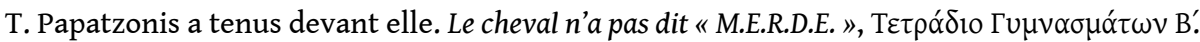

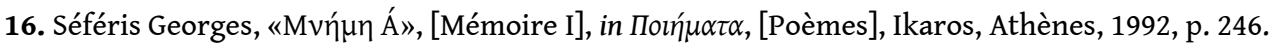

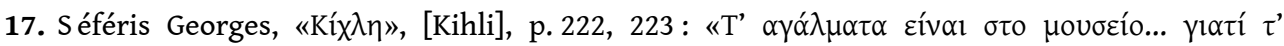

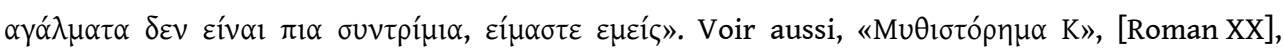

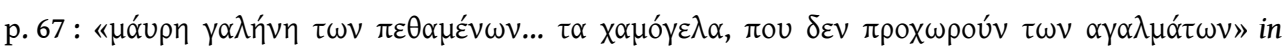
Поюń $\mu \alpha \tau \alpha$, [Poèmes], Ikaros, Athènes 1992.

18. Chant IX., vers 112-115, traduction Victor Bérard, éd. Les Belles Lettres.

19. Le «Petit Paris» que Paul Morand découvre en 1935, se transforme en 1939 pour Georges Séféris comme pour l'écrivain anglais Hector Bolitho, en une capitale qui respire la mort. Voir Catherine Durandin, Histoire des Roumains, Fayard, Paris 1995, p. 84.

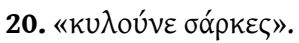

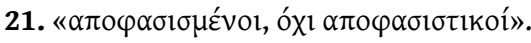

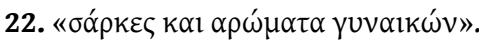

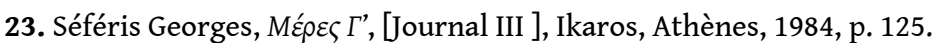

24. Ces mêmes images, le poète les reprendra dans le poème Jours de 1943 que Stratis Tsirkas a associé directement à la révolte de l'armée grecque au Moyen Orient et à la chute de Kanelopoulos. Tsirkas Stratis, «Mı 'A

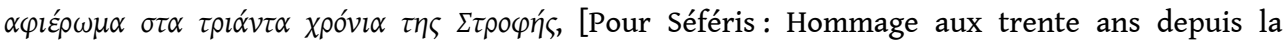
composition de Strophé], Ermis, Athènes 1981 (1961), p. 241-349 (254).

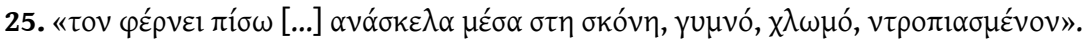

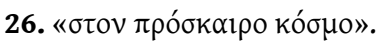

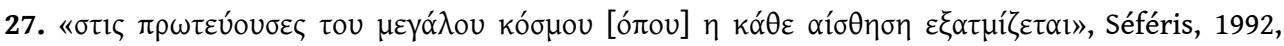
p. 336. Voir aussi Séféris, 2002, p. 128.

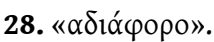

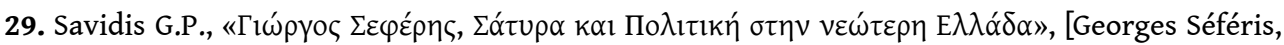

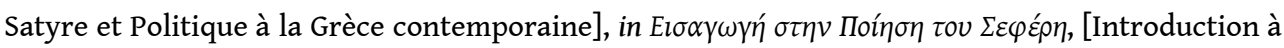
la poésie de Séféris], Panapistimiakes Ekdoseis Kritis, Héraklion 1996, p. 307-340 (320-322).

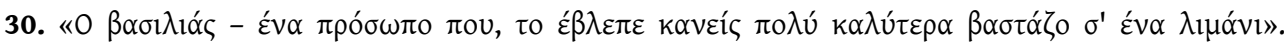
Séféris, 1984, p. 112.

31. «Quel idiot, ce roi ! », en français dans le texte, Séféris, 1984, p. 111.

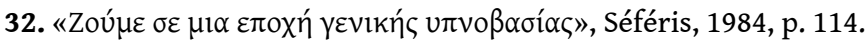

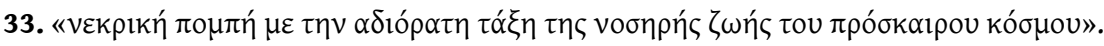

34. Paul Morand semble avoir le même regard que Georges Séféris sur les cérémonies organisées par le roi Carol II. Voir C. Durandin, 1995, p. 283.

35. Savidis, 1996, p. 307-340.

36. Vagenas, 1979, p. 123.

37. Séféris, 1984, p. 111.

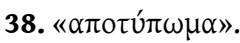




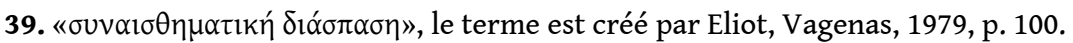

\section{RÉSUMÉS}

Le Cheval de Moldovalachie est le poème que Georges Séféris a composé durant son séjour du 14 ou 29 mai 1939 à Bucarest. Le poète, depuis sa fenêtre de l'Athénée Palace dominant le palais royal et la Calea Victoriei, est comme fasciné par la statue équestre du roi Carol ${ }^{\text {er }}$ qui vient d'être inaugurée - justement le 10 mai 1939, à l'occasion de la célébration du centenaire de sa naissance, en 1839 -, pour célébrer l'avènement du premier roi au trône le 10 mai 1866. Cette statue a vraisemblablement inspiré cette œuvre. Le poète, influencé par le climat angoissant du début de la Seconde Guerre mondiale, transpose ses sentiments négatifs sur la situation politique décadente. Nous nous trouvons au centre politique de la capitale roumaine, au cœur de la ville où symboliquement le pouvoir actuel affronte l'histoire du pays.

The horse of Moldovlahia is the poem that Giorgos Seferis composed during his visit of the 14th to the 29th of May 1939, in Bucharest. The poet is inspired by the equestrian statue of King Charles I that had just been inaugurated-on May 10th 1939-in celebration of his rise to the throne as the first king of Romania, on May 10th 1866, and the centenary of his birth, in 1839. Seferis, influenced by the agonizing atmosphere of the early World War II, transposes his negative emotions to the decadent political situation. We are in the political center of the Romanian capital, the heart of the city where the current power symbolically confronts the country's history.

To 'A

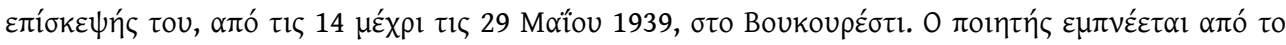

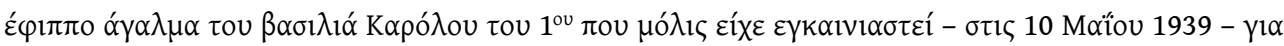

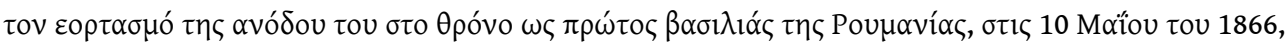

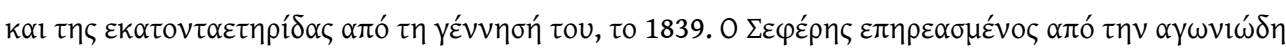

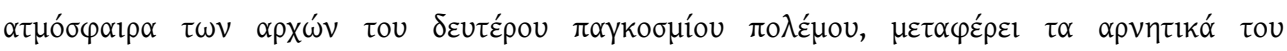

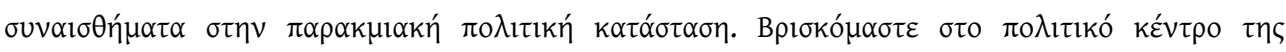

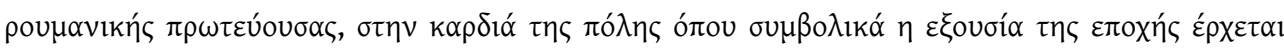

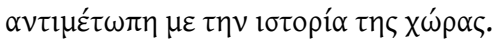


INDEX

Thèmes : Poésie, Littérature grecque

motsclestr Seferis Yorgos (1900-1971), Yunan Romanya, Bükreş, Yirminci yüzyıllarında, Yunan edebiyatı, şiir

Keywords : Seferis Georges (1900-1971), Romania, Bucharest, Twentieth century, Greek

literature, Poetry

Mots-clés : Séféris Giorgos (1900-1971), Séféris Giorgos (1900-1971)

motsclesmk СЕФЕРИС ЈОРГОС (1900-1971), РОМАНИЈА, БУКУРЕШТ, ДВАЕСЕТТИОТ ВЕК, ГРЧКАТА ЛИТЕРАТУРА, ПОЕЗИЈА

Index géographique : Roumanie, Bucarest, Grèce

Index chronologique : vingtième siècle

AUTEUR

GEORGES KOSTAKIOTIS

INALCO - CREE-CEB 